\title{
Impact of machine-learning CT-derived fractional flow reserve for the diagnosis and management of coronary artery disease in the randomized CRESCENT trials
}

\author{
Fay M. A. Nous ${ }^{1,2} \cdot$ Ricardo P. J. Budde ${ }^{1,2} \cdot$ Marisa M. Lubbers ${ }^{1,2} \cdot$ Yuzo Yamasaki $^{3} \cdot$ Isabella Kardys $^{1}$ • \\ Tobias A. Bruning ${ }^{4}$ - Jurgen M. Akkerhuis ${ }^{5} \cdot$ Marcel J. M. Kofflard $^{6} \cdot$ Bas Kietselaer $^{7} \cdot$ Tjebbe W. Galema $^{1}$. \\ Koen Nieman ${ }^{1,2,8}$
}

Received: 6 December 2019 / Revised: 12 February 2020 / Accepted: 25 February 2020

(C) European Society of Radiology 2020

\begin{abstract}
Objective To determine the potential impact of on-site CT-derived fractional flow reserve (CT-FFR) on the diagnostic efficiency and effectiveness of coronary CT angiography (CCTA) in patients with obstructive coronary artery disease (CAD) on CCTA.

Methods This observational cohort study included patients with suspected CAD who had been randomized to cardiac CT in the CRESCENT I and II trials. On-site CT-FFR was blindly performed in all patients with at least one $\geq 50 \%$ stenosis on CCTA and no exclusion criteria for CT-FFR. We retrospectively assessed the effect of adding CT-FFR to the CT protocol in patients with a stenosis $\geq 50 \%$ on CCTA in terms of diagnostic effectiveness, i.e., the number of additional tests required to determine the final diagnosis, reclassification of the initial management strategy, and invasive coronary angiography (ICA) efficiency, i.e., ICA rate without $\geq 50 \%$ CAD.

Results Fifty-three patients out of the 372 patients (14\%) had at least one $\geq 50 \%$ stenosis on CCTA of whom $42 / 53$ patients (79\%) had no exclusion criteria for CT-FFR. CT-FFR showed a hemodynamically significant stenosis $(\leq 0.80)$ in $27 / 53$ patients $(51 \%)$. The availability ofCT-FFR would have reduced the number of patients requiring additional testing by $57 \%$-points compared with CCTA alone ( $37 / 53$ vs. $7 / 53, p<0.001)$. The initial management strategy would have changed for 30 patients $(57 \%, p<0.001)$. Reserving ICA for patients with a CT-FFR $\leq 0.80$ would have reduced the number of ICA following CCTA by $13 \%$-points $(p=0.016)$.

Conclusion Implementation of on-site CT-FFR may change management and improve diagnostic efficiency and effectiveness in patients with obstructive CAD on CCTA.

Key Points

- The availability of on-site CT-FFR in the diagnostic evaluation of patients with obstructive CAD on CCTA would have significantly reduced the number of patients requiring additional testing compared with CCTA alone.

- The implementation of on-site CT-FFR would have changed the initial management strategy significantly in the patients with obstructive CAD on CCTA.

- Restricting ICA to patients with a positive CT-FFR would have significantly reduced the ICA rate in patients with obstructive CAD on CCTA.

Koen Nieman

knieman@stanford.edu

1 Department of Radiology \& Nuclear Medicine, Erasmus University Medical Center, Doctor Molewaterplein 40, 3015

GD Rotterdam, The Netherlands

2 Department of Cardiology, Erasmus University Medical Center, Doctor Molewaterplein 40, 3015 GD Rotterdam, The Netherlands

3 Department of Clinical Radiology, Graduate School of Medical Sciences, Kyushu University, 3-1-1 Maidashi, Higashi Ward, Fukuoka 812-8582, Japan

4 Department of Cardiology, Maasstad Ziekenhuis, Maasstadweg 21, 3079 DZ Rotterdam, The Netherlands

5 Department of Cardiology, Sint Franciscus Gasthuis, Kleiweg 500, 3045 PM Rotterdam, The Netherlands

6 Department of Cardiology, Albert Schweitzer Ziekenhuis, Albert Schweitzerplaats 25, 3318 AT Dordrecht, The Netherlands

7 Department of Cardiology, Zuyderland Medical Center, $\mathrm{H}$. Dunantstraat 5, 6419 PC Heerlen, The Netherlands

8 Stanford University School of Medicine, Cardiovascular Institute, 300 Pasteur Drive, Room H2157, Stanford, CA 94305, USA
\end{abstract}


Keywords Coronary artery disease $\cdot$ Computed tomography angiography $\cdot$ Myocardial fractional flow reserve $\cdot$ Myocardial ischemia $\cdot$ Myocardial perfusion imaging

$\begin{array}{ll}\text { Abbreviations } & \\ \text { CAD } & \begin{array}{l}\text { Coronary artery disease } \\ \text { Coronary computed tomography angiography } \\ \text { CCTA }\end{array} \\ \text { CRESCENT } & \begin{array}{l}\text { Computed Tomography vs. Exercise } \\ \text { Testing in Suspected Coronary Artery Disease } \\ \text { Computed tomography-derived } \\ \text { fractional flow reserve }\end{array} \\ \text { CT-FFR } & \text { Fractional flow reserve } \\ \text { FFR } & \text { Invasive coronary angiography } \\ \text { ICA } & \text { Major adverse cardiovascular events }\end{array}$

\section{Introduction}

Coronary CT angiography (CCTA) has become a recommended diagnostic test in the management of coronary artery disease (CAD) [1]. Although CCTA effectively rules out obstructive $\mathrm{CAD}$, it is limited in the ability to assess the hemodynamic importance of angiographic lesions [2]. Because the anatomic stenosis severity is a weak predictor of hemodynamic significance, functional evaluation is recommended for therapeutic decision-making [3]. CT-derived fractional flow reserve (CT-FFR) can compute FFR values from standard CCTA images without requiring additional testing and radiation exposure, and has shown a good correlation and agreement with invasive FFR in several studies [4-7]. On-site CTFFR software enables CT-FFR analyses on standard workstation without transferring CT images $[8,9]$. Additionally, onsite CT-FFR computation through interpretation of anatomical features (based on machine-learning algorithms) can, in contrary to computational fluid dynamic-based algorithms, be performed in several seconds $[8,10]$. A comprehensive qualitative analysis of CCTA with on-site CT-FFR could provide all essential information for clinical decision-making and reduce the need for further noninvasive and invasive testing. The CRESCENT I and II studies (Computed Tomography vs. Exercise Testing in Suspected Coronary Artery Disease) are pragmatic randomized controlled trials that assessed the clinical effectiveness of a tiered cardiac $\mathrm{CT}$ approach against standard functional testing $[11,12]$. The tiered cardiac CT protocol included a calcium scan, followed by CCTA if calcium was detected. Patients enrolled in CRESCENT II with at least one $\geq 50 \%$ stenosis on CCTA additionally underwent CT myocardial perfusion imaging (CT-MPI). In this study, we performed an on-site CT-FFR analysis using a machinelearning algorithm in patients with suspected CAD and a $\geq 50 \%$ stenosis on CCTA who were enrolled in the CRESCENT I and II cohorts, to assess the potential impact of this functional CCTA application on the diagnostic efficiency and effectiveness of CCTA in patients with obstructive CAD on CCTA.

\section{Methods}

\section{Study population}

This study involved the assessment of CCTA and CT-FFR data from patients who were randomized to cardiac $\mathrm{CT}$ in the CRESCENT I and II trials. The CRESCENT I and II studies are pragmatic randomized controlled trials that assessed the clinical effectiveness of a tiered cardiac CT approach compared with the standard diagnostic work-up using functional testing in patients with suspected CAD. The methods and primary results have been reported previously $[11,12]$. In brief, 618 patients with stable chest pain and suspected CAD were prospectively enrolled in the CRESCENT I and II trials at 6 hospitals in the Netherlands. Patients were randomly assigned to $\mathrm{CT}$ or functional testing in a 2:1 ratio in the CRESCENT I trial and in a 1:1 ratio in the CRESCENT II trial. Exclusion criteria in both trials were a history of known $\mathrm{CAD}$ and an invasive coronary angiography (ICA) or stress test performed $<1$ year ago. Additional exclusion criteria in CRESCENT II were renal impairment, contrast allergy, atrial fibrillation, or other CCTA-specific contraindications. Results of downstream diagnostic and therapeutic procedures were collected from medical records up to 1 year in the CRESCENT I trial and up to 6 months in the CRESCENT II trial. The studies were approved by the medical ethics committees at each participating site, and all participants provided informed consent.

\section{Tiered cardiac CT strategy}

All participants in the cardiac CT group of the CRESCENT trials underwent a non-contrast-enhanced coronary calcium scan (Fig. 1). In patients with a low or intermediate probability of CAD by Diamond and Forrester [13], the absence of calcium excluded obstructive CAD and obviated the need for further testing. Patients with a positive calcium score $(>0)$, as well as patients with a high pre-test probability of CAD (regardless of the calcium score), subsequently underwent CCTA to detect obstructive CAD. In the CRESCENT I trial, patients with a calcium score $>400$ were deferred from CCTA but underwent stress testing or ICA at the discretion of the treating physician. Patients enrolled in the CRESCENT II trial with a $\geq 50 \%$ stenosis on CCTA underwent an adenosine-stress dynamic CT-MPI in the same session. Patients received 
Fig. 1 Cardiac CT algorithm and results. Patient flow diagram of cardiac CT and CT-derived fractional flow reserve (CT-FFR), and its results. High-risk ischemia is defined as 3-vessel disease, left main disease, or proximal left anterior descending coronary disease on coronary $\mathrm{CT}$ angiography (CCTA) and at least one hemodynamically relevant stenosis defined by CT-FFR $\leq 0.80$. CAD = coronary artery disease

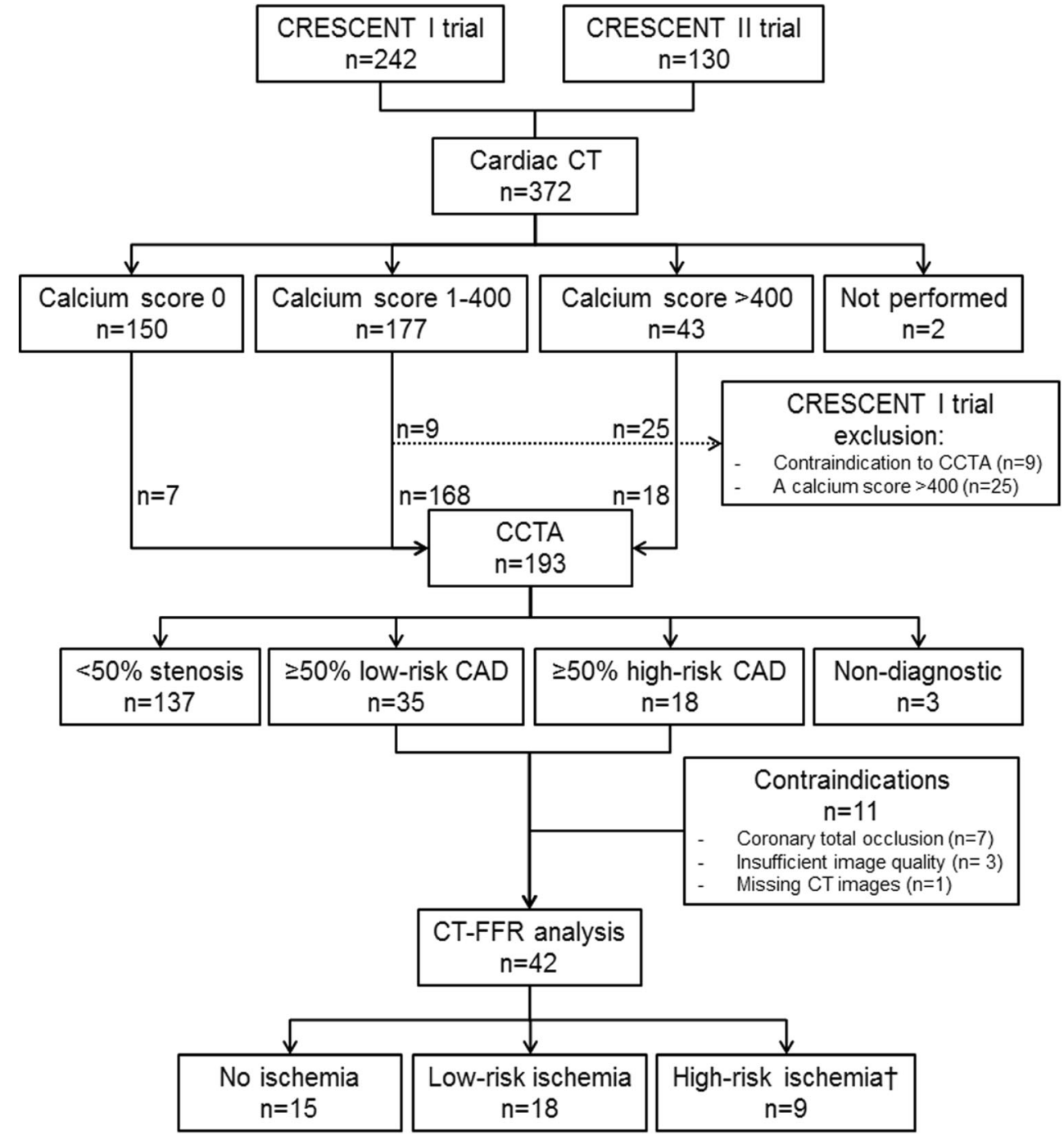

sublingual nitroglycerin before CCTA and if indicated (heart rate $>65 / \mathrm{min}$ ) and clinically acceptable beta-blockers. All sites were equipped with 64-slice or more advanced CT technology. Interpretation of the CT scans combined with available clinical data, as well as subsequent clinical management decisions, was performed by local physicians, in accordance with international guidelines [14].

\section{CT-FFR analysis}

According to European guidelines, functional assessment is recommended in patients with stable chest pain and stenosis $\geq 50 \%$ on CCTA to correlate chest pain symptoms with coronary atherosclerotic ischemia [1]. Therefore, all CCTA examinations performed as part of the CRESCENT trials were reanalyzed with CT-FFR for the purpose of the current study if at least one $\geq 50 \%$ stenosis was present to assess the potential clinical impact of CT-FFR on the diagnostic work-up of CCTA (Table 1; Fig. 1). Exclusion criteria were coronary total occlusion, insufficient image quality, or disrupted CCTA images. Image quality was evaluated based on a 4-point Likert scale: 1 nondiagnostic; 2 impaired image quality, differentiation of coronary artery wall possible with reduced confidence; 3 adequate, reduced image quality due to artifacts without limiting coronary artery wall differentiation; 4 excellent, no artifacts present and clear differentiation of the coronary artery wall. The CT-FFR analysis was performed using a validated machine-learning based CT-FFR software prototype (cFFR version 2.1, Siemens Healthineers; not currently commercially available) [6]. The coronary artery tree was semiautomatically segmented to generate a 3D coronary model. All vessels and side branches of sufficient image quality and a vessel diameter of at least $1.5 \mathrm{~mm}$ were included. The left ventricle myocardial mass was automatically determined from the CT images to estimate the resting total coronary blood flow. Each point on the coronary artery tree was analyzed and CT-FFR was derived based on a combination of pattern recognition and computational learning. Ischemic obstructive 
Table 1 Demographics and baseline patient characteristics
Cardiac CT $(n=372)$

\begin{tabular}{|c|c|}
\hline \multicolumn{2}{|l|}{ Characteristics } \\
\hline Mean age (years) & $56 \pm 11$ \\
\hline Female sex $(\%)$ & $197(53 \%)$ \\
\hline \multicolumn{2}{|l|}{ History $(\%)$} \\
\hline $\begin{array}{l}\text { Transient ischemic attack or } \\
\text { cerebrovascular accident }\end{array}$ & $16(4 \%)$ \\
\hline Peripheral artery disease & $12(3 \%)$ \\
\hline \multicolumn{2}{|l|}{ Cardiac risk factors $(\%)$} \\
\hline Current or past smoker & $125(34 \%)$ \\
\hline Hypertension $^{\mathrm{a}}$ & $192(52 \%)$ \\
\hline Dyslipidemia $^{\mathrm{b}}$ & $179(48 \%)$ \\
\hline Diabetes mellitus $^{\mathrm{c}}$ & $65(17 \%)$ \\
\hline Family history of ischemic heart disease & $139(37 \%)$ \\
\hline Body mass index $\left(\mathrm{kg} / \mathrm{m}^{2}\right) \geq 25^{\dagger}$ & $258(69 \%)$ \\
\hline \multicolumn{2}{|l|}{ Presenting chest pain symptoms (\%) } \\
\hline Typical angina & $106(28 \%)$ \\
\hline Atypical angina & $184(49 \%)$ \\
\hline Non-anginal complaints & $81(22 \%)$ \\
\hline Unknown & $1(<1 \%)$ \\
\hline Pre-test probability - Diamond Forrester $(\%)^{\mathrm{d}}$ & $54(22-79)$ \\
\hline Pre-test probability - genders $(\%)$ & $37(20-58)$ \\
\hline \multicolumn{2}{|l|}{ Medication use $(\%)^{\dagger \dagger}$} \\
\hline Statins or other antiplatelet agents & $172(46 \%)$ \\
\hline Antiplatelets and/or oral anticoagulants & $118(32 \%)$ \\
\hline $\mathrm{ACEi}$ and/or ARB & $77(21 \%)$ \\
\hline Diuretics & $45(12 \%)$ \\
\hline Beta-blockers & $100(27 \%)$ \\
\hline Calcium antagonists & $41(11 \%)$ \\
\hline Nitrates, oral, or sublingual & $22(6 \%)$ \\
\hline
\end{tabular}

Patient characteristics are presented as mean \pm SD, median with 25 th -75 th percentile, and totals with percentages. ${ }^{\mathrm{a}}$ Hypertension: systolic $>150 \mathrm{mmHg}$, diastolic $>90 \mathrm{mmHg}$, or medication use. ${ }^{\mathrm{b}}$ Dyslipidemia: total cholesterol $>5 \mathrm{mmol} / \mathrm{L}$, low-density lipoprotein $>3 \mathrm{mmol} / \mathrm{L}$, or lipid-lowering medication use. ${ }^{c}$ Diabetes mellitus: plasma glucose $>11 \mathrm{mmol} / \mathrm{L}$, or medication use. ${ }^{\mathrm{d}}$ Diamond and Forrester criteria [9]. ${ }^{\dagger}$ Missing data of 7 patients, ${ }^{\dagger \dagger}$ Missing data of 2 patients. ACEi angiotensin-converting-enzyme inhibitors, ARB angiotensin-II receptor blockers
CAD was defined as a stenosis $\geq 50 \%$ on CCTA with a lesionspecific CT-FFR $\leq 0.80$, measured $20 \mathrm{~mm}$ downstream of the stenosis.

\section{Outcomes}

The impact of CT-FFR on clinical management of patients with $\mathrm{a} \geq 50 \%$ stenosis on CCTA was evaluated by comparing the diagnostic efficiency and effectiveness of the observed CCTA strategy with the proposed CT-FFR strategy. The clinical efficiency outcomes included the time to diagnosis from the first outpatient visit until the first test that led to the final diagnosis or the final test that ruled out CAD. Additional tests included all noninvasive tests, including CT-MPI, and ICA to diagnose CAD after the initial tests (calcium scan and/or CCTA). Since CT-FFR was simulated to be part of the CT analysis, results were presumed to be available on the same day as the CCTA results for the diagnostic evaluation. The availability of both anatomical and functional information provided by CCTA and CT-FFR was presumed to result in a final diagnosis and management strategy, which would have made all observed additional tests for determining the final diagnosis or management strategy unnecessary. Initial management strategy consisted of the following options: optimal medical therapy, additional testing required, and revascularization. The CT-FFR-based clinical management decisions were determined based solely on the CCTA and CT-FFR findings, in line with recommendations from international 
guidelines [14]. Information concerning downstream diagnostic testing, as part of the trials or otherwise, as well as clinical outcome data were withheld at the time of CT-FFR-based reassessment of patient management. High-risk obstructive CAD ( $\geq 50 \%$ stenosis) on CCTA, defined as 3-vessel disease, left main disease, or proximal left anterior descending coronary disease, with evidence of ischemia, defined as at least one lesion-specific CT-FFR $\leq 0.80$, was considered an indication for ICA referral in accordance with European guidelines as revascularization is of potential prognostic benefit in those patients [15]. All patients without high-risk obstructive CAD on CCTA or without CT-FFR $\leq 0.80$ were considered eligible for initial medical treatment. Diagnostic effectiveness outcomes included the number of patients having ICA, ICA without $\geq 50 \%$ stenosis, and ICA leading to revascularization. When CT-FFR was not analyzed due to presence of contraindications (coronary total occlusions, insufficient CT image quality, or disrupted CCTA images), outcomes of the observed CCTA strategy and the proposed CT-FFR strategy were presumed to be the same. Major adverse cardiac events (MACE) included death, nonfatal myocardial infarction, unstable angina requiring revascularization, and stroke.

\section{Statistics}

Categorical variables are represented as totals and percentages and continuous variables are represented as means \pm standard deviations or median with 25 th-75th percentiles. McNemar's test and Wilcoxon signed-rank test were used to test the differences in categorical and continuous variables between the observed CT strategy and the proposed CT-FFR strategy. Chisquire test was used to test the differences in ICA leading to revascularization between the observed CT strategy and the proposed CT-FFR strategy. A two-sided $p$ value of $<0.05$ was considered statistically significant. Statistical analyses were performed using SPSS (version 25, IBM Corp).

\section{Results}

\section{Study population}

A total of 370 out of 372 patients $(99 \%)$ randomized to cardiac CT (age $56 \pm 11$ years, 53\% women) had undergone a noncontrast-enhanced coronary calcium scan as their first diagnostic test in the CRESCENT trials (Table 1; Fig. 1). In 150 patients $(41 \%)$, CAD had been excluded based on the absence of coronary calcium and thus had not undergone CCTA as per study protocol. Of the remaining cohort, 193 patients had undergone CCTA, after exclusion of 25 patients with a calcium score $>400$ and 9 patients with contraindications to CCTA (CRESCENT I). CCTA showed $\geq 50 \%$ stenosis in 53 patients $(27 \%)$, including 18 patients $(9 \%)$ with high-risk obstructive
CAD (3-vessel disease, left main disease, or proximal left anterior descending coronary disease).

\section{CT-FFR findings}

The CT-FFR analysis was calculated in $42 / 53$ patients $(79 \%)$ with obstructive CAD ( $\geq 50 \%$ stenosis) on CCTA, as CT-FFR could not be performed in 7 patients with a coronary total occlusion, 3 patients with insufficient CCTA image quality, and 1 patient with a disrupted CCTA dataset (Figs. 1 and 2). CT-FFR $\leq 0.80$ was present in one or more coronary arteries in $27 / 53$ patients $(51 \%)$, including 9 patients $(17 \%)$ with highrisk obstructive CAD on CCTA.

\section{Potential impact of CT-FFR on diagnostic efficiency and medical management}

A total of 54 noninvasive and invasive additional tests, including 29 CT-MPI studies, had been requested in 37/53 patients (70\%) with obstructive CAD on CCTA to reach a final clinical diagnosis and determine the management designation of medical management or revascularization (Fig. 3). In 16 patients (30\%), the final clinical diagnosis had been reached based on CCTA alone and 13 patients had been treated with optimal medical therapy and 3 patients had been directly referred for coronary intervention. CT-FFR was performed in 30 out of 37 patients in whom additional testing had been required to reach the final diagnosis. The availability of CT-FFR in the diagnostic evaluation of patients with obstructive CAD on CCTA would have reduced the number of patients requiring additional testing by $57 \%$-points compared with CCTA alone (from $70 \%(37 / 53)$ to $13 \%(7 / 53), p<0.001)$, and would have rendered $42 / 54(78 \%, p<0.001)$ of the additional tests no longer necessary. This included 18 diagnostic ICAs and 24 noninvasive tests (22 CT-MPI and 2 SPECT studies). Seven patients (13\%) with obstructive CAD would have still required additional testing because CT-FFR could not be performed. Consequently, the implementation of CT-FFR would have reclassified the initial management strategy in 30/53 patients ( $57 \%, p<0.001$ ) with obstructive CAD on CCTA (Fig. 4). Twenty-three patients $(43 \%)$ would have been reclassified from requiring additional testing $(n=17)$ or revascularization $(n=6)$ to optimal medical therapy. Of these patients, ICA had been performed in 12 patients during the course of the CRESCENT trials of whom no one showed high-risk obstructive CAD (3-vessel disease, left main disease, or proximal left anterior descending coronary disease with objective ischemia). Furthermore, CT-FFR would have reclassified 7 patients (13\%) from requiring additional testing to revascularization. Of these patients, ICA had been performed in 6 patients during the course of the CRESCENT trials of which 4 patients showed high-risk obstructive CAD. 
Fig. 2 Case example of CTderived fractional flow reserve. Coronary $\mathrm{CT}$ angiography (CCTA), invasive coronary angiography (ICA), and CT-derived fractional flow reserve (CT-FFR) of a single patient. CCTA showed an intermediate stenosis in the proximal left anterior descending artery, requiring additional assessment to determine management strategy (a). ICA showed a non-obstructive stenosis in the proximal left anterior descending artery (b). The CT-FFR analysis showed no lesion-specific ischemia $($ CT-FFR $>0.80)(\mathbf{c})$

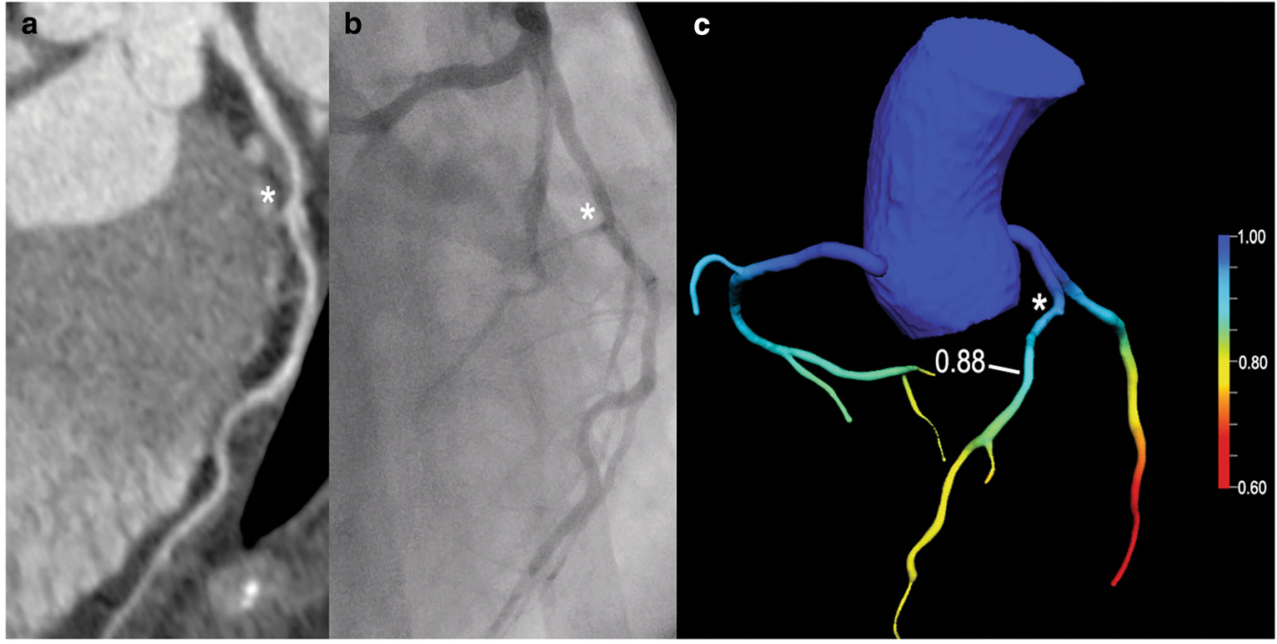

\section{Potential impact of CT-FFR on ICA and revascularization}

At 6-12 months follow-up, 32/53 patients (60\%) with obstructive CAD on CCTA had undergone ICA. Restricting ICA to patients with a positive CT-FFR $(\leq 0.80)$ would have reduced the ICA rate by $13 \%$-points: from $60 \%(32 / 53)$ to $47 \%(25 / 53, p=0.016)$. CT-FFR would have avoided an ICA without $\geq 50 \%$ stenosis in 4 out of 7 patients and would have resulted in a rate of ICA leading to revascularization of $88 \%(22 / 25)$ compared with $78 \%$ (25/32) with CCTA alone $(p=0.487)$. In one patient who did not undergo ICA, an abnormal CT-FFR result would have indicated a need for catheterization/revascularization. Three patients with FFR $>0.80$ were revascularized during the course of the CRESCENT

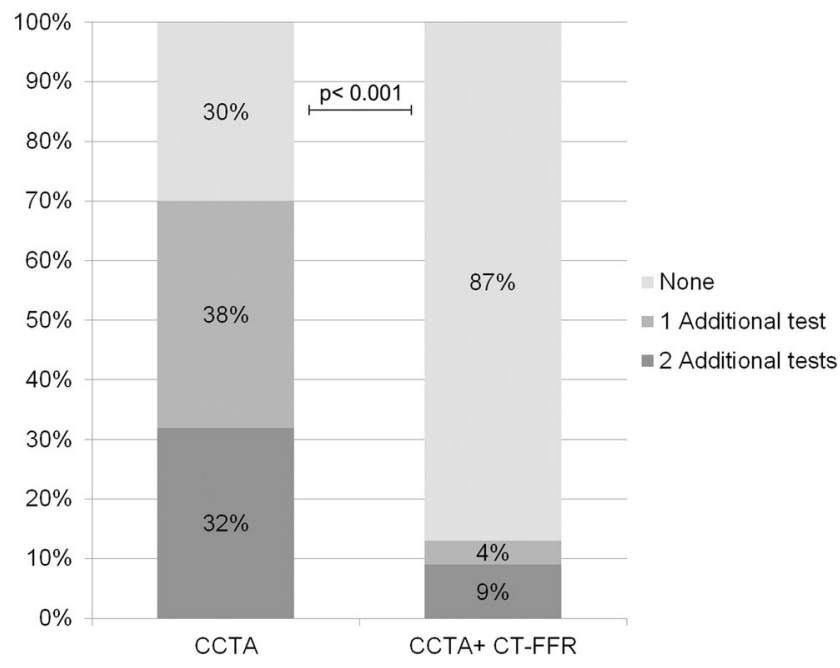

Fig. 3 Proportion of patients requiring additional noninvasive and/or invasive testing for determining management strategy. The proportion of patients that required additional testing to reach the final management designation of medical management or revascularization, observed with the CT strategy and after implementation of CT-derived fractional flow reserve (CT-FFR), divided in none, one, or two additional tests. CCTA = coronary $\mathrm{CT}$ angiography trials; all of whom had low-risk obstructive CAD on CCTA and were initially treated with optimal medical therapy based on CCTA results. One patient had a left bundle branch block on electrocardiography and was referred to ICA after insufficient effect on optimal medical therapy, one patient had a myocardial infarction, and one patient had new angina symptoms 1 year after CCTA.

\section{Safety}

After a median follow-up of 8 (6-12) months, two deaths, three cases of nonfatal myocardial infarctions, two cases of unstable angina requiring revascularization, and no strokes (13\%) had been recorded in 53 patients with obstructive CAD on CCTA. Three events occurred in patients with a CT-FFR $\leq 0.80$ : one non-cardiac death, one nonfatal myocardial infarction associated with medically treated single-vessel CAD detected on CCTA, and one case of unstable angina due to in-stent restenosis. Two nonfatal myocardial infarctions occurred in patients with a CT-FFR >0.80: one patient with medically treated single-vessel CAD detected by CCTA, and one patient without obstructive CAD on CCTA. Two events occurred in patients without performed CT-FFR analyses: one non-cardiac death and one case of unstable angina in a patient with 3-vessel disease diagnosed by CCTA before revascularization was performed.

\section{Comparison between CT-FFR and CT-MPI}

CT-FFR was performed in 22 out of 29 patients (76\%) who underwent CT-MPI as part of the CRESCENT II trial. CTFFR was not calculated in 4 patients with a coronary total occlusion, 2 patients with insufficient CCTA image quality, and 1 patient with a disrupted CCTA dataset. After 612 months follow-up, 11 out of 22 patients underwent ICA of whom 4 patients had high-risk obstructive CAD, 6 patients 
Fig. 4 Flowchart of initial management strategy. Detail breakdown of management allocation by cardiac $\mathrm{CT}$ data alone and after adding CT-derived fractional flow reserve (CT-FFR). ICA = invasive coronary angiography
CCTA

CCTA with CT-FFR

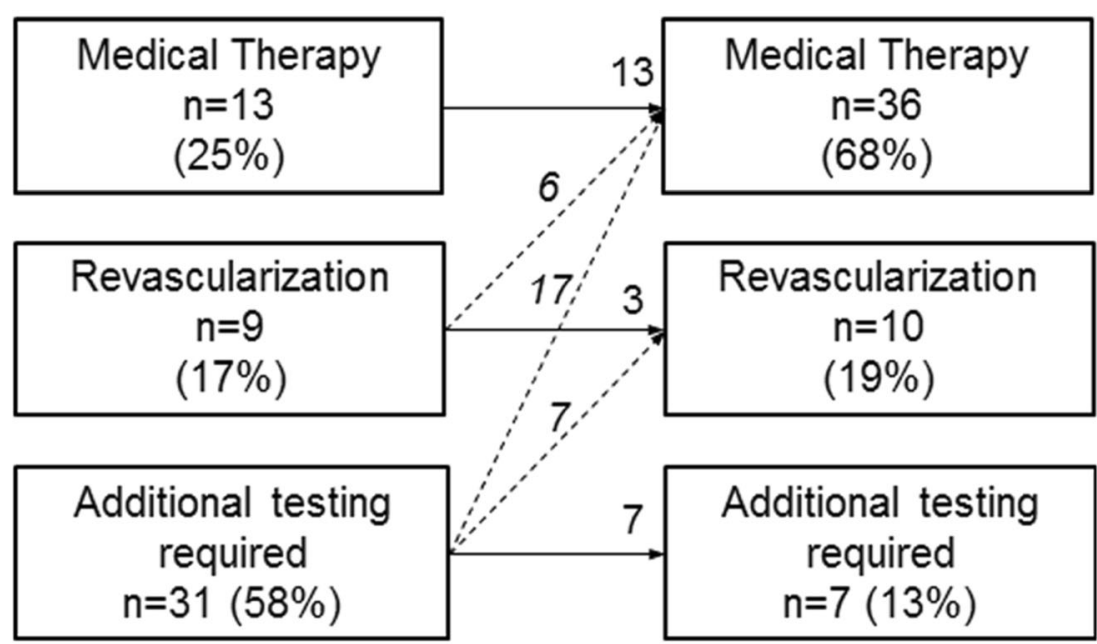

Reclassification rate: $57 \%$

$p<0.001$ had low-risk obstructive $\mathrm{CAD}$, and 1 patient had no obstructive CAD. CT-FFR identified 9 out 10 patients with functionally significant CAD on ICA, whereas CT-MPI identified 8 out of 10 patients. Moreover, CT-FFR correctly identified all 4 patients with high-risk CAD, whereas CT-MPI missed one case and under-classified 2 patients as low-risk CAD $(<10 \%$ left ventricle ischemia).

\section{Discussion}

In this study, we assessed the potential impact of on-site CTFFR on the diagnostic efficiency and effectiveness of a tiered cardiac CT protocol in patients with a stenosis $\geq 50 \%$ on CCTA. We demonstrate that the implementation of CT-FFR may change the diagnostic management, with the potential to expedite the final diagnosis of CAD, diminish the need for additional testing, and reduce the number of ICAs in patients with a stenosis $\geq 50 \%$ on CCTA.

\section{Diagnostic efficiency}

Numerous noninvasive techniques are available for the evaluation of patients with suspect CAD, yet none allows for a complete anatomical and functional assessment of the coronary arteries [14]. Consequently, multiple tests are often required, both noninvasively and invasively, to determine the final diagnosis and management strategy in a population that has a low disease prevalence and benign clinical outcome, as demonstrated by several recent trials $[16,17]$. In the CRESCENT I and II trials, a tiered cardiac CT approach mitigated the cost and burden associated with the diagnostic work-up without limiting the diagnostic performance compared with functional testing [11, 12]. However, CCTA is limited in the ability to determine which patients might benefit most from revascularization, and may result in overtreatment $[5,18,19]$. CT-FFR has the ability to identify a specific coronary lesion that causes ischemia and opens the way to a treatment strategy at a coronary artery lesion-specific level without additional imaging [7]. In this study, we showed that, of the 372 patients who had been randomized to a tiered cardiac CT protocol, only 53 patients (14\%) would have required CT-FFR analysis to decrease the need for additional testing from 19 to merely $11 \%(p<0.001)$ in the entire study population. This demonstrates that the addition of CT-FFR may expedite the diagnosis of $\mathrm{CAD}$ and reduce the need for additional testing. Recently published studies with off-site CTFFR have shown similar results and demonstrated that CTFFR improved the ability of CCTA to identify obstructive CAD in symptomatic patients referred for ICA $[20,21]$. Moreover, there is some evidence to suggest that off-site CTFFR may be cost-effective [22, 23]. However, on-site CT-FFR techniques are not yet approved for clinical use. Overall, these findings support the concept that a combined approach of CCTA and CT-FFR may improve interpretation and evaluation of patients with suspected CAD than CCTA alone.

\section{Medical management}

Hemodynamic evaluation of coronary stenoses using an onsite CT-FFR solution performed by local physicians can reduce diagnostic delay [9]. CT-FFR computation through 
interpretation of anatomical features (based on machinelearning algorithms) can be performed in several seconds with similar diagnostic accuracy as complete coronary flow simulations [6]. This has reduced the overall analysis duration significantly [24]. However, it should be acknowledge that the overall duration of the CT-FFR analysis is determined by the segmentation of the coronary lumen, which strongly depends on disease complexity and image quality $[8,9]$. Current European guidelines recommend additional functional testing in patients with $\geq 50 \%$ stenosis on CCTA; therefore, we restricted the CT-FFR analysis to those with $\geq 50 \%$ stenosis on CCTA [1]. Moreover, it has been suggested that CT-FFR is not of incremental value over CCTA in patients with $<50 \%$ stenosis on CCTA, as the vast majority of these lesions are not hemodynamically significant $[20,25,26]$. However, hemodynamically significant disease cannot completely be ruled out in patients with stenosis $<50 \%$ and CT-FFR could be valuable in specific cases. In our study population, CT-FFR would have reclassified management in more than half of patients with obstructive CAD on CCTA. Our reclassification rate (57\%) was slightly lower than in the prospective, multicenter ADVANCE registry (The Assessing Diagnostic Value of Non-invasive $\mathrm{FFR}_{\mathrm{CT}}$ in Coronary Care), since it incorporated all patients with obstructive CAD on CCTA including the patients without CT-FFR analysis [27]. Nevertheless, our findings might even overestimate the potential effect of CT-FFR since CT-MPI was part of the CRESCENT II study protocol. Our study also showed that the final diagnosis of CAD was determined without any hemodynamic assessment in $30 \%$ of patients with obstructive CAD on CCTA. Since CT-FFR does not require any additional testing and radiation, CCTA with CT-FFR may promote comprehensive anatomically and hemodynamically coronary assessment of patients with suspected CAD, allowing compliance with current recommendations supporting use of both anatomic and functional data in decision-making [15].

\section{Diagnostic effectiveness and safety}

Currently practiced diagnostic strategies based on noninvasive functional testing do not completely achieve their purpose as gatekeeper to the cath lab, which has resulted in a reported low diagnostic yield of ICA [28]. Recently published studies have shown that combining CT-FFR with CCTA could improve the diagnostic yield of ICA and lower ICA rates in patients referred for ICA $[21,29,30]$. Moreover, CT-FFR has shown to be a better predictor for revascularization and MACE compared with CCTA [29, 31, 32]. Our findings represent the first evaluation of on-site CT-FFR application as part of a tiered cardiac $\mathrm{CT}$ protocol and demonstrate that the implementation of CT-FFR may improve the diagnostic yield of ICA and lower ICA rates in patients evaluated for CAD. The ICA rate would have decreased from 12 to $10 \%(p=0.016)$, compared with $13 \%$ in the functional arms of the CRESCENT trials [11, 12]. These results warrant performance of prospective trials to assess the effectiveness and safety of implementing CT-FFR in the management of patients with suspected CAD.

\section{Comparison between CT-FFR and CT-MPI}

CT-MPI allows for visualization or measurement of the regional myocardial blood flow during vasodilator-induced hyperemia. Several studies have shown similar diagnostic performance between CT-MPI and CT-FFR [26, 33, 34]. Also in this cohort, CT-FFR performed at least equally well as CTMPI, but could not be performed in patients with suspected total coronary occlusions or insufficient CCTA imaging quality. More research is needed to establish the respective value of each respective technique, or potentially combined in a tiered diagnostic approach to assess patients with suspected CAD.

\section{Limitations}

The observational nature of this study implies that the CT-FFR findings were not available to the physicians and did not affect clinical decision-making. We can only project, based on international guidelines, how physicians would have applied CTFFR results had they been available. Additionally, we used an existing dataset from two trials with minor protocol differences in order to test our current hypothesis. CT-MPI was indicated by the CRESCENT II study protocol and could potential overestimate the potential impact of CT-FFR. ICA data was not available in all patients in these pragmatic clinical trials.

In conclusion, our study demonstrates that the implementation of on-site CT-FFR in a tiered cardiac CT protocol improved the diagnostic efficiency and effectiveness in patients with obstructive CAD on CCTA.

Acknowledgments We owe gratitude to all participating patients of the CRESCENT trials, as well as the medical teams, in particular Paul Musters, who made this study and the CRESCENT trials possible.

Funding information This study has received funding by grants from the Dutch Heart Foundation (NHS 2014T061 and NHS 2013T071).

\section{Compliance with ethical standards}

Guarantor The scientific guarantor of this publication is Koen Nieman.

Conflict of interest The authors of this manuscript declare relationships with the following companies: Siemens Healthineers, HeartFlow, GE Healthcare, Bayer Healthcare.

Statistics and biometry Isabella Kardys kindly provided statistical advice for this manuscript. 
Informed consent Written informed consent was obtained from all subjects (patients) in this study.

Ethical approval Institutional Review Board approval was obtained.

Study subjects or cohorts overlap All subjects have been previously reported in the CRESCENT I and CRESCENT II trials. The prior studies evaluated the clinical effectiveness of a tiered cardiac CT approach against standard functional testing. The current study expands on these studies by combing the subjects of the CT arm of both studies and implement CT-FFR analyses into the protocol. The previous studies were published in European Heart Journal (CRESCENT I trial) and JACC Cardiovascular Imaging (CRESCENT II trial)

\section{Methodology}

- Retrospective

- Observational

- Multicenter study

\section{References}

1. Knuuti J, Wijns W, Saraste A et al (2019) 2019 ESC Guidelines for the diagnosis and management of chronic coronary syndromes: the Task Force for the diagnosis and management of chronic coronary syndromes of the European Society of Cardiology (ESC). Eur Heart J. https://doi.org/10.1093/eurheartj/ehz425

2. Budoff MJ, Nakazato R, Mancini GB et al (2016) CT angiography for the prediction of hemodynamic significance in intermediate and severe lesions: head-to-head comparison with quantitative coronary angiography using fractional flow reserve as the reference standard. JACC Cardiovasc Imaging 9:559-564

3. Tonino PA, De Bruyne B, Pijls NH et al (2009) Fractional flow reserve versus angiography for guiding percutaneous coronary intervention. N Engl J Med 360:213-224

4. Norgaard BL, Leipsic J, Gaur S et al (2014) Diagnostic performance of noninvasive fractional flow reserve derived from coronary computed tomography angiography in suspected coronary artery disease: the NXT trial (analysis of coronary blood flow using CT angiography: next steps). J Am Coll Cardiol 63:1145-1155

5. von Knebel Doeberitz PL, De Cecco CN, Schoepf UJ et al (2019) Coronary CT angiography-derived plaque quantification with artificial intelligence CT fractional flow reserve for the identification of lesion-specific ischemia. Eur Radiol 29:2378-2387

6. Coenen A, Kim YH, Kruk M et al (2018) Diagnostic accuracy of a machine-learning approach to coronary computed tomographic angiography-based fractional flow reserve: result from the MACHINE consortium. Circ Cardiovasc Imaging 11:e007217

7. Zhuang B, Wang S, Zhao S, Lu M (2020) Computed tomography angiography-derived fractional flow reserve (CT-FFR) for the detection of myocardial ischemia with invasive fractional flow reserve as reference: systematic review and meta-analysis. Eur Radiol 30: $712-725$

8. Tesche C, De Cecco CN, Baumann S et al (2018) Coronary CT angiography-derived fractional flow reserve: machine learning algorithm versus computational fluid dynamics modeling. Radiology 288(1):64-72. https://doi.org/10.1148/radiol.2018171291

9. Coenen A, Lubbers MM, Kurata A et al (2016) Coronary CT angiography derived fractional flow reserve: methodology and evaluation of a point of care algorithm. J Cardiovasc Comput Tomogr 10: $105-113$

10. Itu L, Rapaka S, Passerini T et al (2016) A machine-learning approach for computation of fractional flow reserve from coronary computed tomography. J Appl Physiol (1985) 121(1):42-52. https://doi.org/10.1152/japplphysiol.00752.2015

11. Lubbers M, Dedic A, Coenen A et al (2016) Calcium imaging and selective computed tomography angiography in comparison to functional testing for suspected coronary artery disease: the multicentre, randomized CRESCENT trial. Eur Heart J 37:12321243

12. Lubbers M, Coenen A, Kofflard M et al (2017) Comprehensive cardiac CT with myocardial perfusion imaging versus functional testing in suspected coronary artery disease: the multicenter, randomized CRESCENT-II trial. JACC Cardiovasc Imaging 11(11): 1625-1636. https://doi.org/10.1016/j.jcmg.2017.10.010

13. Diamond GA, Forrester JS (1979) Analysis of probability as an aid in the clinical diagnosis of coronary-artery disease. N Engl J Med 300:1350-1358

14. Task Force Members, Montalescot G, Sechtem U et al (2013) 2013 ESC guidelines on the management of stable coronary artery disease: the Task Force on the management of stable coronary artery disease of the European Society of Cardiology. Eur Heart J 34: 2949-3003

15. Neumann FJ, Sousa-Uva M, Ahlsson A et al (2019) 2018 ESC/ EACTS Guidelines on myocardial revascularization. EuroIntervention 14:1435-1534

16. Douglas PS, Hoffmann U, Patel MR et al (2015) Outcomes of anatomical versus functional testing for coronary artery disease. $\mathrm{N}$ Engl J Med 372:1291-1300

17. Scot-Heart Investigators (2015) CT coronary angiography in patients with suspected angina due to coronary heart disease (SCOTHEART): an open-label, parallel-group, multicentre trial. Lancet 385:2383-2391

18. Meijboom WB, Meijs MF, Schuijf JD et al (2008) Diagnostic accuracy of 64-slice computed tomography coronary angiography: a prospective, multicenter, multivendor study. J Am Coll Cardiol 52: 2135-2144

19. Budoff MJ, Dowe D, Jollis JG et al (2008) Diagnostic performance of 64-multidetector row coronary computed tomographic angiography for evaluation of coronary artery stenosis in individuals without known coronary artery disease: results from the prospective multicenter ACCURACY (Assessment by Coronary Computed Tomographic Angiography of Individuals Undergoing Invasive Coronary Angiography) trial. J Am Coll Cardiol 52:1724-1732

20. Curzen NP, Nolan J, Zaman AG, Norgaard BL, Rajani R (2016) Does the routine availability of CT-derived FFR influence management of patients with stable chest pain compared to CT angiography alone?: the FFRCT RIPCORD study. JACC Cardiovasc Imaging 9: 1188-1194

21. Douglas PS, Pontone G, Hlatky MA et al (2015) Clinical outcomes of fractional flow reserve by computed tomographic angiographyguided diagnostic strategies vs. usual care in patients with suspected coronary artery disease: the prospective longitudinal trial of FFR(CT): outcome and resource impacts study. Eur Heart J 36: 3359-3367

22. Nielsen LH, Olsen J, Markenvard J, Jensen JM, Norgaard BL (2013) Effects on costs of frontline diagnostic evaluation in patients suspected of angina: coronary computed tomography angiography vs. conventional ischaemia testing. Eur Heart J Cardiovasc Imaging 14:449-455

23. Hlatky MA, De Bruyne B, Pontone G et al (2015) Quality-of-life and economic outcomes of assessing fractional flow reserve with computed tomography angiography: PLATFORM. J Am Coll Cardiol 66:2315-2323

24. Baumann S, Hirt M, Schoepf UJ et al (2019) Correlation of machine learning computed tomography-based fractional flow reserve with instantaneous wave free ratio to detect hemodynamically significant coronary stenosis. Clin Res Cardiol. https://doi.org/10. 1007/s00392-019-01562-3 
25. Gaur S, Ovrehus KA, Dey D et al (2016) Coronary plaque quantification and fractional flow reserve by coronary computed tomography angiography identify ischaemia-causing lesions. Eur Heart J 37:1220-1227

26. Hamon M, Geindreau D, Guittet L, Bauters C, Hamon M (2019) Additional diagnostic value of new CT imaging techniques for the functional assessment of coronary artery disease: a meta-analysis. Eur Radiol 29:3044-3061

27. Fairbairn TA, Nieman K, Akasaka T et al (2018) Real-world clinical utility and impact on clinical decision-making of coronary computed tomography angiography-derived fractional flow reserve: lessons from the ADVANCE Registry. Eur Heart J 39:3701-3711

28. Patel MR, Peterson ED, Dai D et al (2010) Low diagnostic yield of elective coronary angiography. N Engl J Med 362:886-895

29. Lu MT, Ferencik M, Roberts RS et al (2017) Noninvasive FFR derived from coronary $\mathrm{CT}$ angiography: management and outcomes in the PROMISE Trial. JACC Cardiovasc Imaging 10: $1350-1358$

30. Jensen JM, Botker HE, Mathiassen ON et al (2018) Computed tomography derived fractional flow reserve testing in stable patients with typical angina pectoris: influence on downstream rate of invasive coronary angiography. Eur Heart J Cardiovasc Imaging 19:405-414

31. Patel MR, Norgaard BL, Fairbairn TA et al (2019) 1-year impact on medical practice and clinical outcomes of FFRCT: the ADVANCE Registry. JACC Cardiovasc Imaging 13(1 Pt 1):97-105. https://doi. org/10.1016/j.jcmg.2019.03.003

32. Norgaard BL, Hjort J, Gaur S et al (2017) Clinical use of coronary CTA-derived FFR for decision-making in stable CAD. JACC Cardiovasc Imaging 10:541-550

33. Coenen A, Rossi A, Lubbers MM et al (2017) Integrating CT myocardial perfusion and CT-FFR in the work-up of coronary artery disease. JACC Cardiovasc Imaging 10:760-770

34. Pontone G, Baggiano A, Andreini D et al (2019) Stress computed tomography perfusion versus fractional flow reserve $\mathrm{CT}$ derived in suspected coronary artery disease: the PERFECTION study. JACC Cardiovasc Imaging 12:1487-1497

Publisher's note Springer Nature remains neutral with regard to jurisdictional claims in published maps and institutional affiliations. 